\title{
Symmetrical Distribution of Primes and Their Gaps
}

\author{
Brandon Y. Wang1, Xin Wang2 \\ ${ }^{1}$ School of Mechanical, Electrical \& Information Engineering, Shandong University, Weihai, China \\ ${ }^{2}$ State Key Laboratory of Palaeobiology and Stratigraphy, Nanjing Institute of Geology and Palaeontology and Center for \\ Excellence in Life and Paleoenvironment, Chinese Academy of Sciences, Nanjing, China \\ Email: xinwang@nigpas.ac.cn
}

How to cite this paper: Wang, B.Y. and Wang, X. (2021) Symmetrical Distribution of Primes and Their Gaps. Advances in Pure Mathematics, 11, 447-456.

https://doi.org/10.4236/apm.2021.115031

Received: March 29, 2021

Accepted: May 22, 2021

Published: May 25, 2021

Copyright (c) 2021 by author(s) and Scientific Research Publishing Inc. This work is licensed under the Creative Commons Attribution-NonCommercial International License (CC BY-NC 4.0). http://creativecommons.org/licenses/by-nc/4.0/

\begin{abstract}
Primes are of great importance and interest in mathematics partially due to their hard-to-predict distribution. A corollary of the Goldbach Conjecture is that two primes are equally distanced from a mid-point integer. Here the authors demonstrate that most primes are bilateral symmetrically distributed on the both sides of the halves of super products (or their integer multiples) of primes. This pattern suggests that greater primes may be obtained more efficiently by subtracting smaller ones from constants equal to super products (or their integer multiples) of primes.
\end{abstract}

\section{Keywords}

Prime Number, Distribution, Bilateral Symmetry, Super Product, Pairwise

\section{Introduction}

Primes appear to distribute randomly and they catch much attention from mathematicians for long time [1]-[11]. The Goldbach Conjecture states that every even number greater than 4 is a sum of two primes. A corollary of the conjecture is that every single integer greater than 3 is equally distanced from two primes, implying that at least two primes are paired on both sides of an integer. This pattern has been documented in previous publications [10] [12] [13]. However, how many prime pairs there are on both sides of an integer remains to be an unanswered question. Trying to answer this question, we found that more primes tend to be paired on both sides of the halves of super products (or their integer multiples) of primes. Many mathematicians have noted the existence of prime gaps as well as twin primes (which have a difference of 2 in between) [5] [14], but whether there is any regularity about the occurrence of 
such gaps remains an open question. Here we demonstrate that the pairwise (bilateral symmetrical) distributions of primes and their gaps near the super products (or its integer multiples), hoping it will trigger more interesting investigations.

\section{Methods}

Initially, the target of our investigation was to figure out how many prime pairs have the same sum. The statistics indicated pair number peaks at super products of primes (or their integer multiples). We analyzed and proved the rationality underlying these peaks, and proved the existence of gaps around super products of primes (or their integer multiples) and validated a routine generating primes.

\section{Results}

After we manually obtained number of prime pairs for every even number under 220 (Table 1) and did statistics (Figure 1), it became obvious that there are local peaks at $30,60,90,120 \ldots$, namely, the number peaks periodically.

\section{Theoretical Analysis and Proof}

The $n$th prime is denoted as $P_{n}$. The product of the first $n-1$ primes is designated as Super Product of $P_{n}$, and denoted as $X_{n}$ [9].

Theorem 1. There are at most two primes, namely, $X_{n}-1$ and $X_{n}+1$, in $\left(X_{n}-\right.$ $\left.P_{n}, X_{n}+P_{n}\right)$, and these two, if both valid, constitute twin primes.

Proof.

1) By definition, $X_{n}$ is a composite.

2) Since $a \mid X_{n}$ and $a \nmid 1$ for all $a \in\left\{P_{i} \mid 1 \leq i<n\right\}$, therefore $a \nmid\left(X_{n}-1\right)$ and $a \nmid\left(X_{n}+1\right)$. As $\left(X_{n}+1\right)=\left(X_{n}-1\right)+2$, so if both $\left(X_{n}-1\right)$ and $\left(X_{n}+1\right)$ are primes, they constitute twin primes.

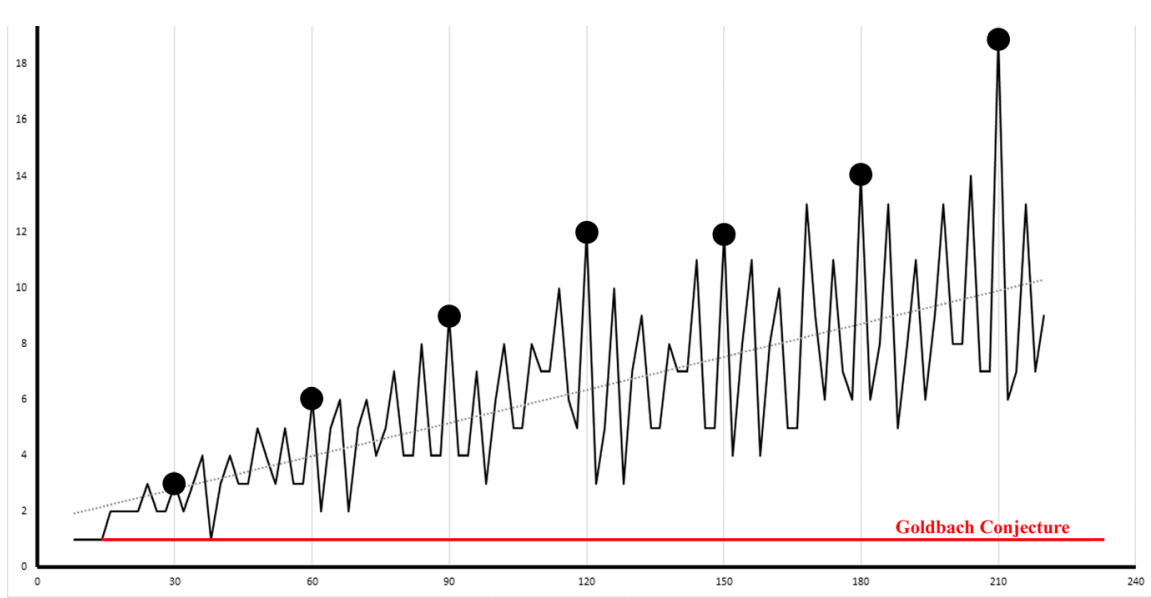

Figure 1. Number of prime pairs for each even number under 220 demonstrates a general increasing trend, and it peaks periodically at the multiples of 30 . Note that all the numbers are equal to or greater than 1 , the number required by the Goldbach Conjecture. 
Table 1. The first 107 even numbers and the paired primes.

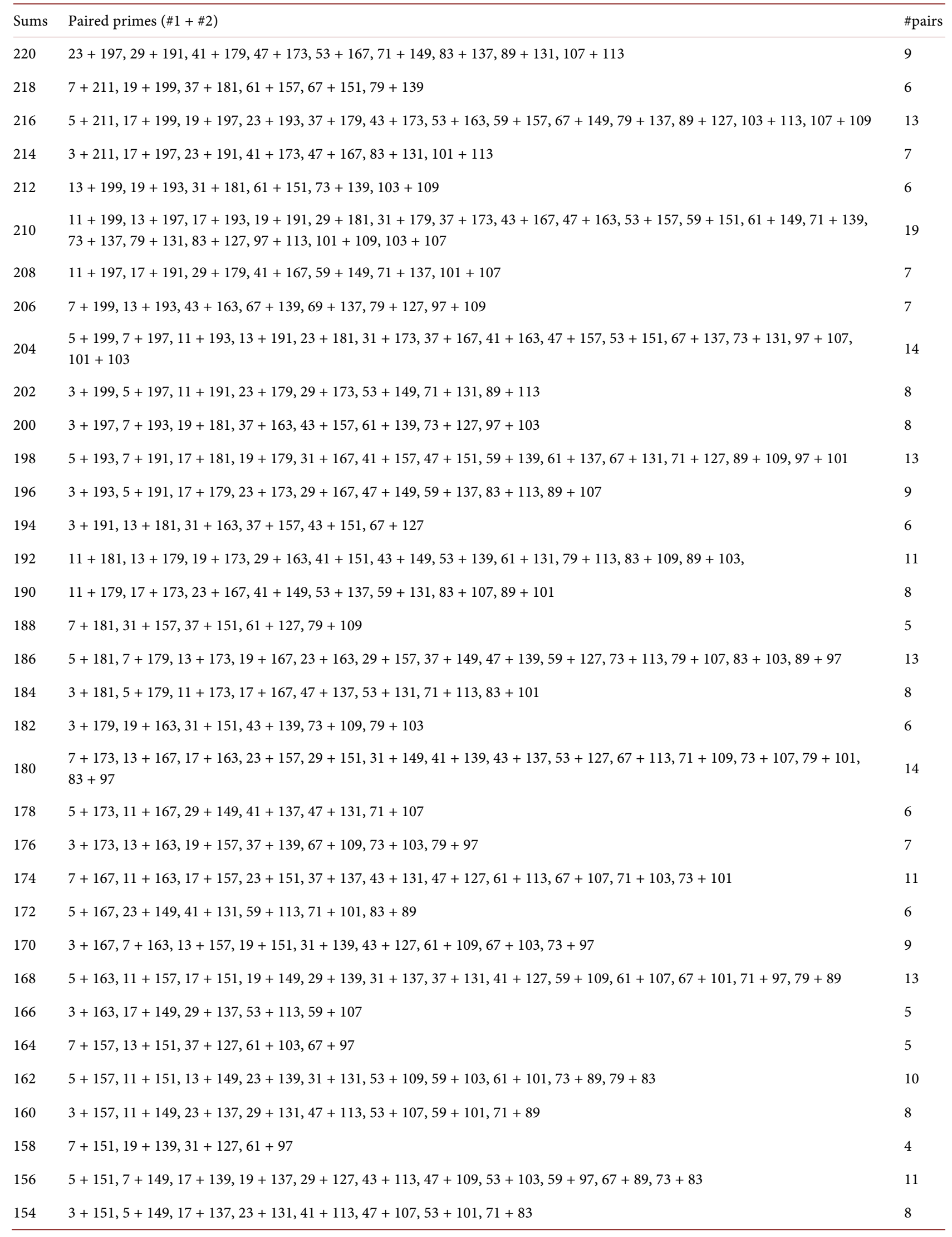




\section{Continued}

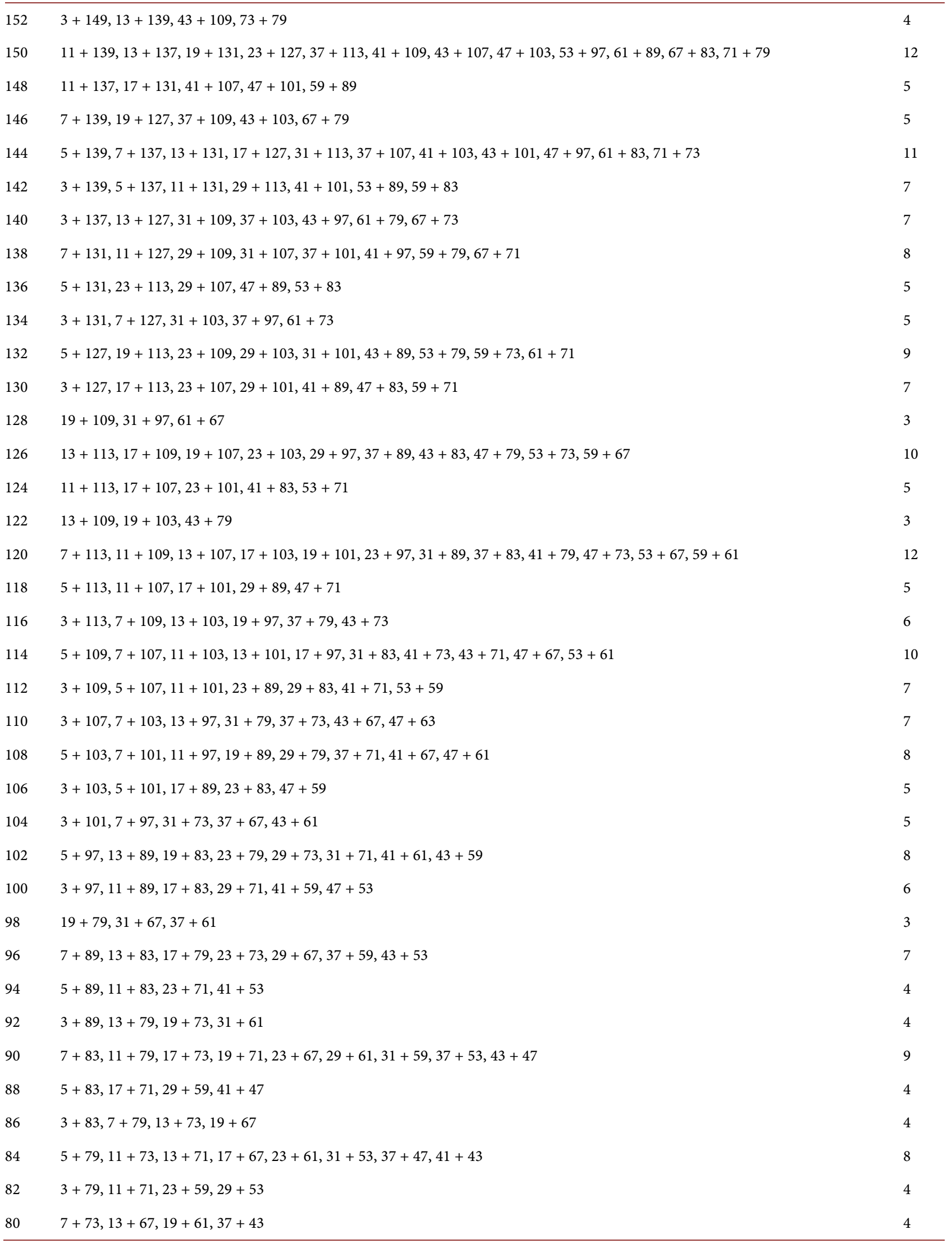




\section{Continued}

\begin{tabular}{|c|c|c|}
\hline 78 & $5+73,7+71,11+67,17+61,19+59,31+47,37+41$ & 7 \\
\hline 76 & $3+73,5+71,17+59,23+53,29+47$ & 5 \\
\hline 74 & $3+71,7+67,13+61,31+43$ & 4 \\
\hline 72 & $5+67,11+61,13+59,19+53,29+43,31+41$ & 6 \\
\hline 70 & $3+67,11+59,17+53,23+47,29+41$ & 5 \\
\hline 68 & $7+61,31+37$ & 2 \\
\hline 66 & $5+61,7+59,13+53,19+47,23+43,29+37$ & 6 \\
\hline 64 & $3+61,5+59,11+53,17+47,23+41$ & 5 \\
\hline 62 & $3+59,19+43$ & 2 \\
\hline 60 & $7+53,13+47,17+43,19+41,23+37,29+31$ & 6 \\
\hline 58 & $5+53,11+47,17+41$ & 3 \\
\hline 56 & $3+53,13+43,19+37$ & 3 \\
\hline 54 & $7+47,11+43,13+41,17+37,23+31$ & 5 \\
\hline 52 & $5+47,11+41,23+29$ & 3 \\
\hline 50 & $3+47,7+43,13+37,19+31$ & 4 \\
\hline 48 & $5+43,7+41,11+37,17+31,19+29$ & 5 \\
\hline 46 & $3+43,5+41,17+29$ & 3 \\
\hline 44 & $3+41,7+37,13+31$ & 3 \\
\hline 42 & $5+37,11+31,13+29,19+23$ & 4 \\
\hline 40 & $3+37,11+29,17+23$ & 3 \\
\hline 38 & $7+31$ & 1 \\
\hline 36 & $5+31,7+29,13+23,17+19$ & 4 \\
\hline 34 & $3+31,5+29,11+23$ & 3 \\
\hline 32 & $3+29,13+19$ & 2 \\
\hline 30 & $7+23,11+19,13+17$ & 3 \\
\hline 28 & $5+23,11+17$ & 2 \\
\hline 26 & $3+23,7+19$ & 2 \\
\hline 24 & $5+19,7+17,11+13$ & 3 \\
\hline 22 & $3+19,5+17$ & 2 \\
\hline 20 & $3+17,7+13$ & 2 \\
\hline 18 & $5+13,7+11$ & 2 \\
\hline 16 & $3+13,5+11$ & 2 \\
\hline 14 & $3+11$ & 1 \\
\hline 12 & $5+7$ & 1 \\
\hline 10 & $3+7$ & 1 \\
\hline 8 & $3+5$ & 1 \\
\hline
\end{tabular}

3) Since $a \mid X_{n}$ and $a \mid a$ for all $a \in\left\{P_{i} \mid 1 \leq i<n\right\}$, therefore $a \mid\left(X_{n}-a\right)$ and $a \mid\left(X_{n}+a\right)$, namely, all $X_{n}-a$ and $X_{n}+a$ are composites for all 
$a \in\left\{P_{i} \mid 1 \leq i<n\right\}$.

4) If $a$ is a composite smaller than $P_{n}$, a must have all of its prime factors $b \in\left\{P_{i} \mid 1 \leq i<n\right\}$. Since $b \mid X_{n}$ and $b \mid a$, therefore $b \mid\left(X_{n}-a\right)$ and $b \mid\left(X_{n}+a\right)$, therefore all $X_{n}-a$ and $X_{n}+a$ are composites.

In summary, $X_{n}-1$ and $X_{n}+1$ are the only numbers in $\left(X_{n}-P_{n}, X_{n}+P_{n}\right)$ that can be primes and with a difference of 2 in between.

This completes the proof.

Note. $X_{n}-1$ and $X_{n}+1$ does not necessarily be a prime, either of them may be divided exactly by a prime equal to or greater than $P_{n}$.

In case none of $X_{n}-1$ and $X_{n}+1$ is a prime, $\left(X_{n}-P_{n}, X_{n}+P_{n}\right)$ is a $2 P_{n}$ long prime gap. An interesting inference is "As $P_{n}$ approaches to the infinite, length of the gap also approaches the infinite". This answers the Question vii asked by Dr. Hua on page 90 of his book [15]. Considering the known greatest prime is more than 24 million digits long [6], it is amazing to conceive that there exists such a long gap of primes: at most only two primes are immersed in zillions and zillions of composites! This provides one solution for the problem of prime gap (A8) and raw material for hypotheses on difference between consecutive primes mentioned in [4] [11].

Assuming $X_{n}+1$ and $X_{n}+P_{n}$ both are primes, let's try to search for the next prime after $X_{n}$. Starting from $X_{n}$, the next number is $X_{n}+1$ which is a prime. We finish the search with only one test, with $100 \%$ success rate. Starting from $X_{n}+2$, we cannot succeed until reaching $X_{n}+P_{n}$. The success rate is $2 /\left(P_{n}-2\right)$ if we only test all the odds in the range. This rate decreases as the value of $P_{n}$ grows greater. Knowing the above knowledge about prime gap, the test can be restricted to all odds in $\left[X_{n}+P_{n-1}+1, X_{n}+P_{n}\right]$, the success rate is $2 /\left(P_{n}-P_{n-1}\right)$ (Figure 2).

Theorem 2. For $a \in\left\{P_{i} \mid i \geq n\right\}$ and $b \in\left\{P_{i} \mid 1 \leq i<n\right\}, b$ cannot divide $X_{n}$ a exactly.

Proof.

Since $b \mid X_{n}$ and $b \nmid a$, therefore $b \nmid\left(X_{n}-a\right)$.

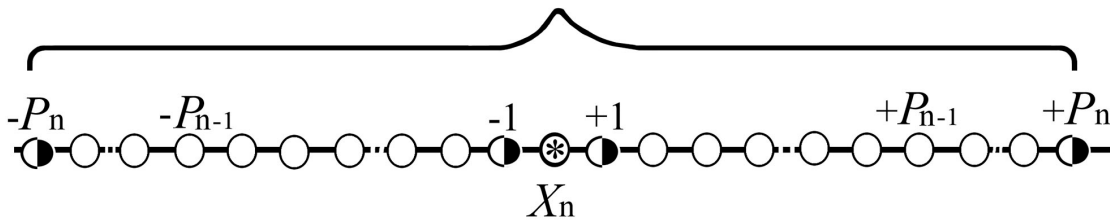

$\bigcirc \begin{gathered}\text { Composite } \\ \text { number }\end{gathered} \underset{\text { product }}{\text { Super }} \begin{gathered}\text { Composite/prime } \\ \text { number }\end{gathered}$

Figure 2. Prime gap $\left(X_{n}-P_{n}, X_{n}+P_{n}\right)$ centered around $X_{n}$. Note the primality of $X_{n}-1$ and $X_{n}+1$ determines length of the gap: if both are composites, the gap is $2 P_{n}$ long; if both are primes, they are the only primes in $\left(X_{n}-P_{n}, X_{n}+P_{n}\right)$ and they are twin primes, and the lengths of the gap are reduced to $P_{n}-1$; if one of them is a prime, then the other is the only prime in $\left(X_{n}-P_{n}, X_{n}+P_{n}\right)$, and the lengths of the gap are reduced to $P_{n}-1$ and $P_{n}+1$, respectively. A few examples of such gaps are $(23,37),(53,67),(83$, 97), (113, 127), (143, 157), (173, 187), (199, 221) with 29 and 31, 59 and 61, 89, none, 149 and 151, 179 and 181,211, respectively, as prime exceptions. 
This completes the proof.

Note. This does not necessarily mean that $X_{n}-a$ is a prime, as it may be divided exactly by a prime equal or greater than $P_{n}$. This is the shortcoming of this paper, namely, we cannot eliminate the all influence of numbers greater than $P_{n}$. However, it does imply that $X_{n}-a$ is very likely a prime. This constitutes the rationality underlying the bilateral symmetrical distribution of primes shown in Figures 3-5.

\section{Implications on Relationship between Primes}

As implied by Theorem 2, primes can be paired under certain condition. Now we designate $S$ as a constant equal to $X_{n}$ (or its integer multiples), then $S / 2$ can be a mid-point integer between of prime pairs on its both sides. The relationship between such prime pairs is termed complementary here since the sums of such pairs are always equal to a constant $S$, as shown in Figures 3-5.

Note 1 . Although 1 is not a prime, its complementary may be a prime.

Note 2. Some of the numbers in $(S / 2, S)$ obtained by subtracting smaller primes may be composites. These exceptions can be eliminated case-by-case by calculating all combination products of all primes in $\left[P_{n}, S / P_{n}\right]$ : if any of their products falls in $\left(P_{m}, S / 2\right)$, add the complementary of the product into the list; if any of their products falls in $(S, S / 2)$, delete the product from the generated list.

Note 3. As shown in Figure 3, when $S=30, P_{n}=7, S / P_{n}=4$, the range $\left[P_{n}\right.$, $\left.S / P_{n}\right]$ becomes [7,4], which is an empty range. This explains the lack of exceptions in Figure 3 although such exceptions occur in Figure 4 and Figure 5.

\section{Algorithm Generating Primes}

Taking advantage of the above described pairing relationship between primes, routine generating greater primes includes the following steps (using Figure 3 as an example).

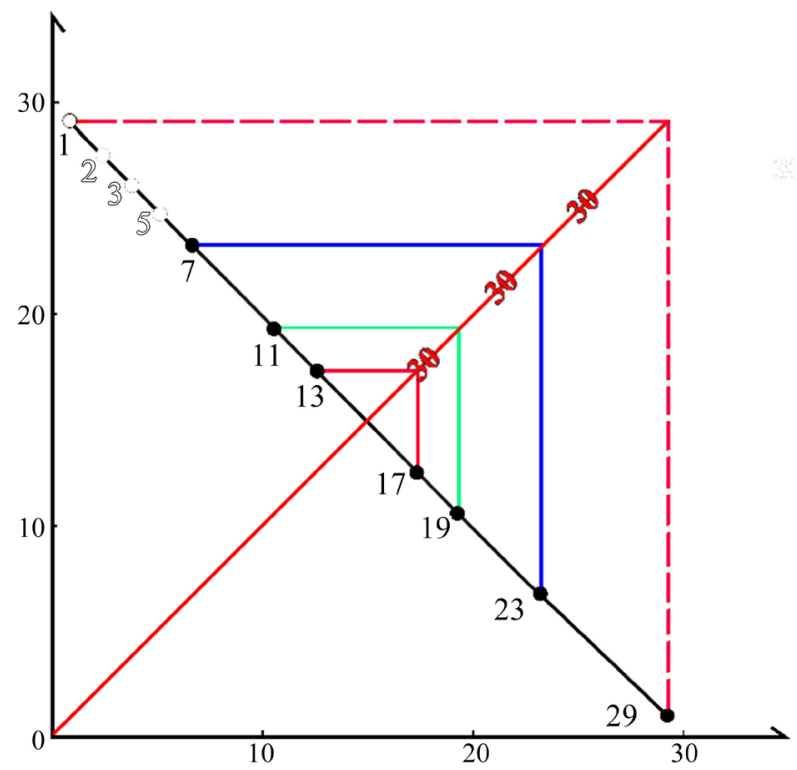

Figure 3. Prime pairs with sums equal to 30 , with no exception. 


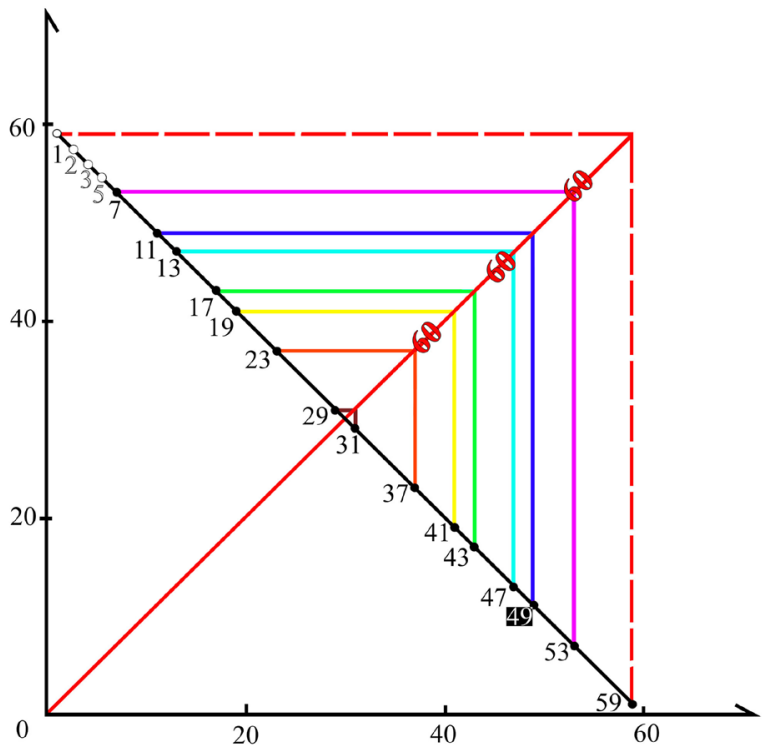

Figure 4. Prime pairs with sums equal to 60 , with one exception of $49(=7 \times 7)$.

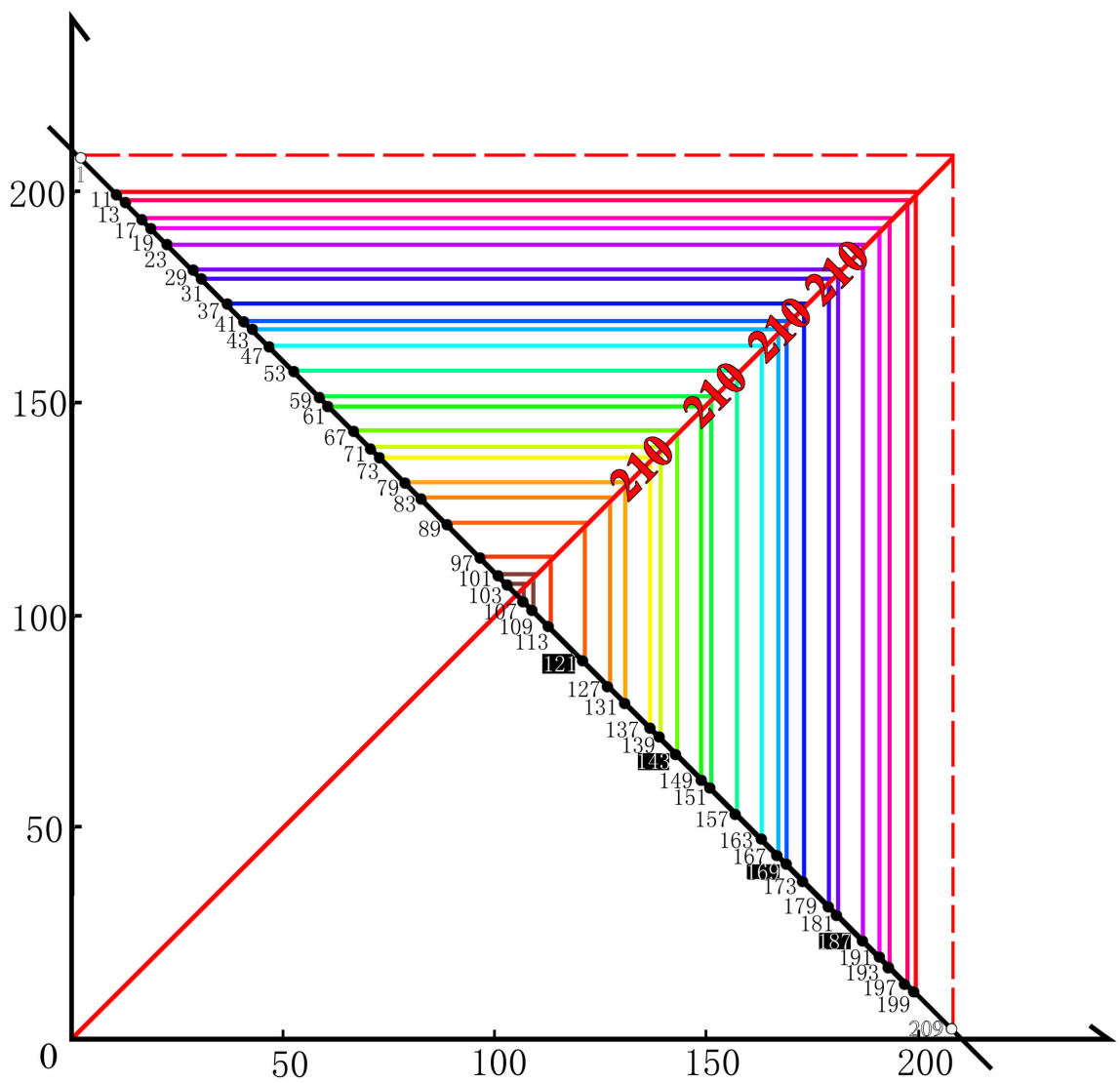

Figure 5. Prime pairs with sums equal to 210 , with four exceptions of $121(=11 \times 11), 143$ $(=11 \times 13), 169(=13 \times 13)$, and $187(=11 \times 17)$.

Step 1. Calculate the value of $S$ ( $X_{n}$ or its integer multiples).

Step 2. Subtract 1 and all primes in $\left[P_{n}, S / 2\right)$ from $S$, save the result in an ascending order as a list. 
Step 3. Calculate all combination products in $\left(P_{n}, S\right)$ of all primes in $\left[P_{n}, S / P_{n}\right]$. If such a product is within the list obtained in Step 2, delete it from the list.

Step 4 . Save the above generated list. Finish.

\section{Discussions}

Compared to the existing routines generating primes, the present one has the following advantages:

1) The calculation involved is computationally cheap. The candidate list of greater primes can be obtained by subtracting smaller ones from a constant.

2) The result is dense, namely, all primes within scope are covered.

3) Although the applicable range of each run is limited, the applicable range of the routine can be extended exponentially into the infinite, as it is hinged with super products of primes.

\section{Conclusion}

Primes tend to be pairwise distributed. Such pairing relationship implies that greater primes can be obtained in a computationally cheap way. There is either one continuous $2 P_{n}$ long prime gap or two at least $P_{n}-1$ long prime gaps around $X_{n}$. One or two of $X_{n}-1$ and $X_{n}+1$ may be the only primes within $\left(X_{n}-P_{n}, X_{n}+\right.$ $\left.P_{n}\right)$.

\section{Acknowledgements}

This research was supported by the Strategic Priority Research Program (B) of Chinese Academy of Sciences (Grant No. XDB26000000), and National Natural Science Foundation of China (41688103, 91514302). We appreciate the constructive suggestions from two anonymous reviewers and Mr. Wuwei Wang.

\section{Conflicts of Interest}

The authors declare no conflicts of interest regarding the publication of this paper.

\section{References}

[1] Devlin, K. (1999) Mathematics: The New Golden Age. Columbia University Press, New York.

[2] Luque, B. and Lacasa, L. (2009) The First-Digit Frequencies of Prime Numbers and Riemann Zeta Zeros. Proceedings of the Royal Society A, 564, 2197-2216. https://doi.org/10.1098/rspa.2009.0126

[3] Oliver, R.J.L. and Soundaranjan, K. (2016) Unexpected Biases in the Distribution of Consecutive Primes. PNAS, 113, E4446-E4454.

https://doi.org/10.1073/pnas.1605366113

[4] Zhang, Y. (2013) Bounded Gaps between Primes. Annals of Mathematics, 179, 1121-1174. https://doi.org/10.4007/annals.2014.179.3.7

[5] Sha, Y.Y. (2017) Twin Prime Number Theorem. Global Journal of Science Frontier Research, 17, 41-45. 
[6] GIMPS (2018) GIMPS Discovers Largest Known Prime Number: $2^{82,589,933}-1$. https://www.mersenne.org/primes/?press=M82589933

[7] Zhao, G., You, J., Sun, J. and Yin, G. (2015) Further Discussion of Prime Number Distribution Rule. Henan Science, 33, 506-508.

[8] Li, B. (2001) Distribution Rule of Prime Numbers. Journal of Jiaozuo University, 4, 45-46. https://doi.org/10.16214/j.cnki.cn41-1276/g4.2001.04.012

[9] Wang, X. (2021) The Genesis of Prime Numbers-Revealing the Underlying Periodicity of Prime Numbers. Advances in Pure Mathematics, 11, 12-18.

https://scirp.org/journal/paperinformation.aspx?paperid=106566 https://doi.org/10.4236/apm.2021.111002

[10] Cai, T. (2019) Figurate Primes and Goldbach Conjecture. Advances in Mathematics (China), 48, 766-768.

[11] Guy, R.K. (2007) Unsolved Problems in Number Theory. Science Press, Beijing.

[12] Ghanouchi, J. (2013) About an Even as the Sum or the Difference of Two Primes. Bulletin of Mathematical Science and Applications, 5, 35-43. https://doi.org/10.18052/www.scipress.com/BMSA.5.35

[13] Qiu, Y. (2017) Symmetry Distribution Law of Prime Numbers of Positive Integers and Related Results. International Journal of Science and Engineering Applications, 6, 360-363. https://core.ac.uk/display/105342972

[14] Aiazzi, B., Baronti, S., Santurri, L. and Selva, M. (2019) An Investigation on the Prime and Twin Prime Number Functions by Periodical Binary Sequences and Symmetrical Runs in a Modified Sieve Procedure. Symmetry, 11, 775. https://doi.org/10.3390/sym11060775

[15] Hua, L.-G. (1979) Introduction to Number Theory. Science Press, Beijing. 